computed. Remarkably, the information content is as large as in a numerical simulation - but this is an experiment! The packing fraction can be varied from $\phi=0.597$ to $\phi=0.773$ by changing the number of beads. The jamming transition has been estimated in earlier work ${ }^{9}$ to take place at $\phi \cong 0.83$.

The authors measure the evolution of all the dynamical characteristics, from the mean square displacement to observables related to dynamical heterogeneity as a function of the packing fraction. In particular, they compute the average size of mobile clusters (which turn out to be formed by strings as in supercooled liquids), the average length of these strings and, from a four-point function, the length over which the relaxation dynamics is correlated. All of these quantities, as well as the relaxation timescales, increase on approaching the jamming transition.

This is a major result. It is a strong indication that the jamming transition may really be a phase transition - although of a new kind, because no growing static long-range order is detected. Only dynamical correlations increase approaching the transition. The authors fit the growth of the time- and length scales using laws that were introduced for glass-forming liquids: the Vogel-Fulcher-Tammann (VFT) law and power laws. Remarkably, the VFT fit gives a transition value, $\phi_{\mathrm{VFT}} \cong 0.84$, which is very close to the one independently determined for the jamming transition'.

It is a mark of the value of this study that it raises at least as many questions as it resolves. Indeed, the fits presented by the authors, although not conclusive, are very suggestive. Could it be that explanations of the glass transition work for the jamming transition? More generally, if jamming is indeed a critical phenomenon, what is the nature of the underlying phase transition? Contrary to the case for crystalline solids, could the emergence of rigidity be the driving mechanism of the transition, as has been proposed ${ }^{10,11}$ ? In this case, would a small but finite drive and a non-zero temperature (or deformation of the grains) transform the transition into a crossover? Or could the divergence of length and timescales be instead related to a bona fide thermodynamic transition towards a static amorphous long-range order, as proposed for the glass transition ${ }^{12}$ and for hardsphere systems ${ }^{13}$ ?

These and many other questions remain. But the experimental study of the critical properties of the jamming transition, initiated in the work of Keys et al. ${ }^{2}$, offers a promising route to answering at least some of them.

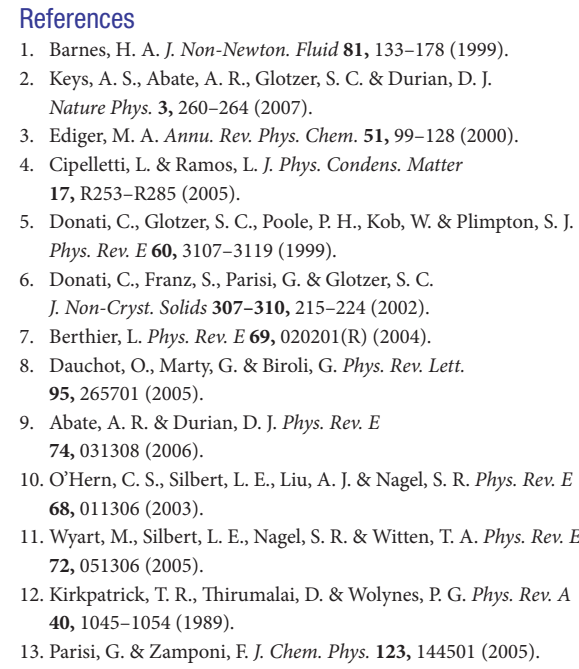

References

keys, A. S., Abate, A. R., Glotzer, S. C. \& Durian, D.

17, R253-R285 (2005)

\& Plimpton, S. J.

. Dauchot, O. Marty G. \& Biroli, G. Phys. Rev. Lett.

95, 265701 (2005)

68, 011306 (2003)

.

Kirkpatrick, T. R.,

3. Parisi, G. \& Zamponi, F. J. Chem. Phys. 123, 144501 (2005).

\title{
CHEMICAL PHYSICS
}

\section{How many kisses?}

Isaac Newton and David Gregory couldn't agree on how many identical spheres can simultaneously touch a central sphere of the same radius: 12 , said Newton, 13 Gregory. It was only long after their deaths that Newton was rigorously proved right. In most dimensions higher than three, however, this 'kissing-number' problem is still unsolved, and so is the case, even in three dimensions, in which the to-bekissed sphere has a different radius from those of the spheres surrounding it. Andreas Hermann and colleagues have considered a related problem in chemistry (Angew. Chem. Int. Edn 46, 2444-2447; 2007), searching for the highest possible coordination number for a given species - that is, the highest number of ligands that can be packed into one shell around a central atom with which they all interact (but without having strong interactions between the ligands).

In many problems, a coordination number, $N$, of 12 leads to stable configurations, such as clusters with icosahedral structures, or the face-centred cubic and hexagonal

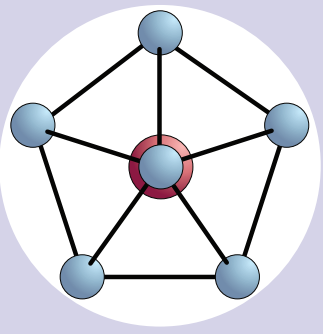

close-packed lattices in solids. However, the firstprinciples quantum-chemical simulations by Hermann et al. indicate that for the case of a single charged lead atom surrounded by helium atoms, up to 15 ligands fit in one coordination sphere. This would make $\mathrm{PbHe}_{15}^{2+}$ the first gas-phase molecule that has a coordination number higher than 12 . The authors present evidence that the molecule is stable, and expect that the new record holder should be identifiable by mass spectrometry.

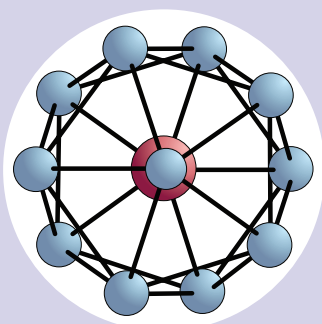

The series of $\mathrm{PbHe}_{N}^{2+}$ molecules goes through shapes of all kinds as the number of helium atoms is increased (see pictures from top left). Up to $N=6$, all of the ligands lie in one half-sphere, but are then equally distributed when the ligands number 8 to $12 ;(N=7$, however, looks a bit like an umbrella). Hermann et al. found that they can comfortably push further, up to $N=15$, and still accommodate all of the helium atoms in one shell.

But, for more than 15 ligands, it seems that the central lead atom rejects the intimacy of kissing, preferring to keep further adherers at some distance in a second coordination shell (final picture).

Andreas Trabesinger 